\title{
Extraction of Backscattering and Absorption Coefficients of Magnetite Nanosphere Composites from Light-Scattering Measurements: Implications for Optomagnetic Sensing
}

\author{
Junxin Wang, Changgang Xu,* Hui Xiong, Yuanyuan Han, Annica M. Nilsson, Mattias Strömberg, \\ Tomas Edvinsson, and Gunnar A. Niklasson*
}

Cite This: ACS Appl. Nano Mater. 2020, 3, 11172-11183

Read Online

ACCESS | Lلll Metrics \& More | 国 Article Recommendations | s supporting Information

ABSTRACT: Interaction of light with magnetic nanoparticles, dispersed in solution or embedded in other materials, is of major interest in a range of applications, one example being optomagnetic sensors. In applied research, light absorption and scattering of nanoparticle composites are often quantified by the Kubelka-Munk two-flux radiative transfer model. In this paper, we synthesized magnetite $\left(\mathrm{Fe}_{3} \mathrm{O}_{4}\right)$ nanospheres with different diameters and encapsulated them into a polymer matrix. Their spectral transmittance and reflectance were investigated by spectrophotometry,

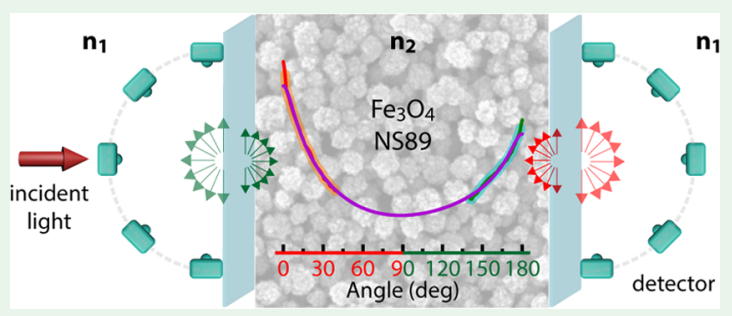
together with measurements of angle-resolved scattering in the forward and

backward hemispheres. The measured angular distribution was applied to approximate the scattering-phase function inside the film, which could be well described by the revised Reynolds-McCormick model. The backscattering and absorption coefficients were derived by inversion of the Kubelka-Munk relations, using the interface reflectances obtained from angle-dependent measurements. We present detailed optical properties for samples with various particle concentrations and scattering layer thicknesses, consisting of the magnetite and polymer composites. The absorption and backscattering coefficients for particles of diameter $458 \mathrm{~nm}$ showed qualitative agreement with single-scattering Mie calculations. The optical properties of composites with smaller particles might be influenced by an oxidized $\mathrm{Fe}_{2} \mathrm{O}_{3}$-like surface layer. The present approach can be used to study different kinds of magnetic nanoparticle clusters, dispersed in a supporting medium, and thus provide optical parameters of relevance for interpreting results of optomagnetic sensing experiments.

KEYWORDS: iron oxide, Kubelka-Munk, angle-resolved scattering, backscattering and absorption coefficients, radiative transfer, nanoparticles, Mie simulation

\section{INTRODUCTION}

The physical properties of magnetic colloidal nanoparticles are of interest for a number of innovative applications. ${ }^{1,2}$ Novel synthesis, self-assembly, and functionalization methods have led to advances, for example, in the biomedical field. ${ }^{3,4}$ In particular, the possibility to detect variations in optical properties due to an external magnetic field has attracted considerable interest. ${ }^{5}$ Magnetic nanoparticles dispersed in a surrounding medium exhibit unique optical performance in, for example, optical capacitors, ${ }^{6}$ volumetric particle receivers and reactors for photothermal and thermochemical processes, ${ }^{7,8}$ direct absorption solar collectors, ${ }^{9}$ and magnetically induced formation of photonic nanostructures. ${ }^{10-13}$ In the field of biomedical applications, iron-oxide particles are of special interest since they can be controlled by external magnetic fields, are biocompatible, have a large surface-to-volume ratio, and can be modified by functional biomolecules on the surface. $^{14,15}$ Applications range from bioseparation, photothermal cancer treatment, and drug delivery to magnetic biosensors. ${ }^{4,16}$ In particular, magnetic particle biosensors have unique advantages such as cost-efficiency, user-friendliness, and a low background signal; ${ }^{17}$ also a readout signal can be detected by either magnetic or optical methods. ${ }^{18}$ The latter type of sensors are called optomagnetic sensors and several detection principles using homogeneous readouts (volumebased sensing) have been developed. ${ }^{18-20}$ Most of these methods rely on the possibility to manipulate clusters and chains of magnetic nanoparticles by changing the direction of, or rotating, an applied magnetic field, thereby inducing optical variations. Analyte molecules are able to bind to the particles, thereby increasing the tendency for chain or cluster formation and hence producing a measurable optical signal.

Received: August 26, 2020

Accepted: October 22, 2020

Published: November 11, 2020

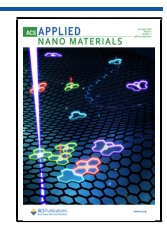


To develop a fundamental understanding of the optomagnetic sensing process, knowledge of the parameters characterizing optical absorption and scattering of sizedependent magnetic nanoparticles is needed. In a solid or liquid medium containing particles with sizes comparable to the wavelength of the incident light, light scattering cannot be ignored. In such a case, the optical properties can be described by radiative transfer theory (RTT). ${ }^{21}$ In this paper, we are concerned with the inverse problem of RTT, i.e., to obtain absorption and scattering coefficients for the studied material from experimental transmittance and reflectance spectra. Inversion methods based on numerical solutions of the RTT are computationally challenging but the inverse addingdoubling (IAD) 22,23 and inverse Monte-Carlo (IMC) methods $^{24,25}$ have been frequently used for this purpose. These methods rely on accurate numerical solutions of the RTT and are able to obtain single-particle volumetric absorption and scattering cross sections as well as a scattering asymmetry parameter, without further approximations. These cross sections are directly proportional to the absorption and scattering coefficients of the material. When particle clustering is important, dependent scattering effects within the clusters are significant, and this is clearly so for optomagnetic sensing materials. In this case, the IAD and IMC methods provide equally accurate absorption and scattering coefficients from the effective cross sections of the clusters. Another, approximate, approach to the solution of the RTT is given by the phenomenological two- and four-flux models for light scattering, which do not implicitly include single-particle properties. The four-flux model would be preferable as the foundation for an inversion scheme because of its close correspondence to Monte-Carlo simulations, ${ }^{26,27}$ but a reliable inversion scheme does not exist at present. ${ }^{28}$ The KubelkaMunk (KM) two-flux radiative transfer model ${ }^{29,30}$ is a useful approximation, which has been widely used in applied studies to characterize pigmented coatings, ${ }^{31}$ paints, ${ }^{32}$ and paper. $^{33}$ The two-flux model takes into account one diffuse flux of the radiation field propagating in the forward direction and one diffuse flux traveling in the backward direction, in a planeparallel layer. The theory has been generalized to total (the sum of diffuse and collimated) fluxes using well-controlled approximations in the four-flux theory. ${ }^{34}$ In addition, robust methods to invert the KM equations to obtain absorption and scattering coefficients have been developed. ${ }^{35,36}$ However, in the KM model, additional parameters, i.e., interface reflectances, are often poorly known. Frequently, the assumption of an isotropic scattering pattern ${ }^{34,35}$ has been used to compute these parameters but this limits the ability to describe anisotropic scattering. Therefore, a thorough characterization of the angular scattering patterns ${ }^{37}$ is essential to apply the $\mathrm{KM}$ model to experimental data in a more rigorous way.

Here, we present a detailed experimental investigation of the wavelength-dependent and angle-dependent optical properties of magnetite $\left(\mathrm{Fe}_{3} \mathrm{O}_{4}\right)$ nanoparticles with different diameters encapsulated into a polymer matrix. We measured the optical transmittance and reflectance in conjunction with the angledependent light scattering of the nanoparticle composite layer. The angle-dependent information is further used to derive the scattering-phase function inside the layer. This information was essential to determine interfacial reflectances occurring in the KM theory. Subsequently, the absorption and backscattering coefficients were computed by fitting the KM theory to experimental transmittance and reflectance data. The resulting absorption and backscattering coefficients were compared to simulations by the Mie theory. Moreover, a detailed study of the dependence of the optical parameters of the nanocomposites as a function of particle concentration and layer thickness was carried out.

\section{RESULTS AND DISCUSSION}

2.1. Sample Preparation and Characterization. The $\mathrm{Fe}_{3} \mathrm{O}_{4}$ nanoparticles were prepared by a wet chemistry approach and their diameters were determined to be $89 \pm$ $19 \mathrm{~nm}$ (NS89), $205 \pm 27 \mathrm{~nm}$ (NS205), and $458 \pm 137 \mathrm{~nm}$ (NS458) from scanning electron microscopy (SEM) images (Figure 1a-f). The SEM images showed that these synthesized

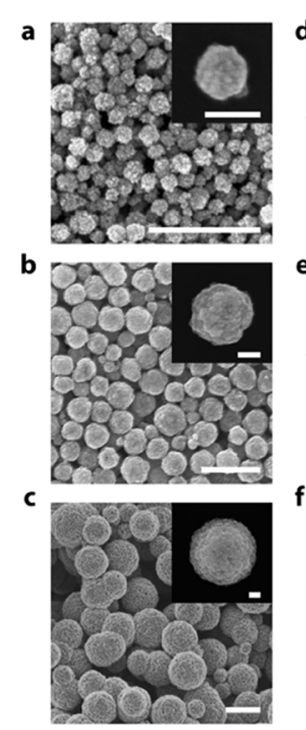

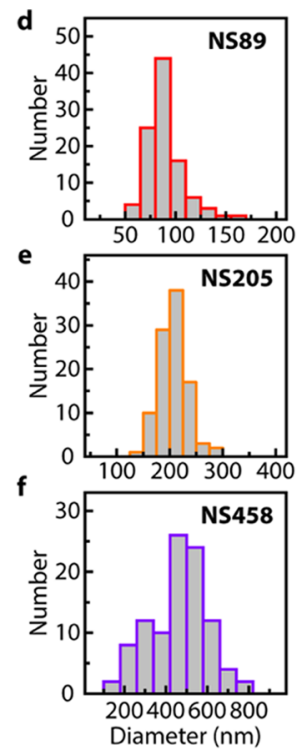

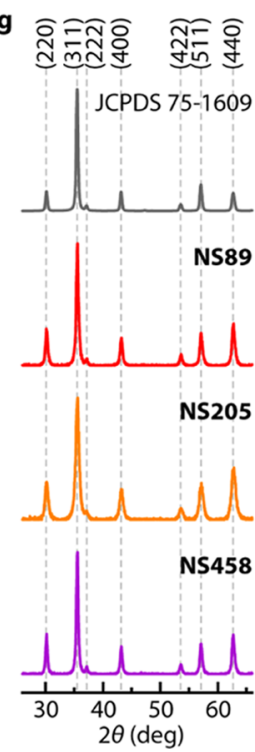

Figure 1. $(a-c)$ Scanning electron microscope (SEM) images (scale bars $=500 \mathrm{~nm}$ ) with zoom-in images of single particles (inset scale bars $=100 \mathrm{~nm}) .(\mathrm{d}-\mathrm{f})$ Size distributions of synthesized $\mathrm{Fe}_{3} \mathrm{O}_{4}$ nanoparticles with diameters of $89 \pm 19 \mathrm{~nm}$ (NS89), $205 \pm 27 \mathrm{~nm}$ (NS205), and $458 \pm 137 \mathrm{~nm}$ (NS458). (g) X-ray diffraction (XRD) patterns of the three $\mathrm{Fe}_{3} \mathrm{O}_{4}$ samples with the patterns indexed with reference to fcc magnetite (JCPDS card 75-1609).

$\mathrm{Fe}_{3} \mathrm{O}_{4}$ particles were close to spherical (less spherical for smaller particles) with rough surfaces (insets of Figure $1 \mathrm{a}-\mathrm{c}$ ). $\mathrm{X}$-ray diffraction (XRD) patterns (Figure $1 \mathrm{~g}$ ) indicated that the nanospheres were highly crystalline. The XRD patterns were in excellent agreement with the face-centered cubic (fcc) structure of magnetite $\mathrm{Fe}_{3} \mathrm{O}_{4}$ (JCPDS card no. 75-1609). No $\mathrm{Fe}_{2} \mathrm{O}_{3}$ phase could be detected by XRD. The magnetization curves were recorded by a vibrating sample magnetometer (VSM, Section 4) and showed a clear ferromagnetic behavior (Supporting Information, Figure S1) with a magnetization reaching over $60 \mathrm{~A} \cdot \mathrm{m}^{2} \cdot \mathrm{kg}^{-1}$ at room temperature, when the maximum applied field was $800 \mathrm{kA} \cdot \mathrm{m}^{-1}$, for the three types of particles. The samples showed no hysteresis loops. The saturation magnetization is $92 \mathrm{~A} \cdot \mathrm{m}^{2} \cdot \mathrm{kg}^{-1}$ for $\mathrm{Fe}_{3} \mathrm{O}_{4}, 0.5 \mathrm{~A}$. $\mathrm{m}^{2} \cdot \mathrm{kg}^{-1}$ for $\alpha-\mathrm{Fe}_{2} \mathrm{O}_{3}$, and $84 \mathrm{~A} \cdot \mathrm{m}^{2} \cdot \mathrm{kg}^{-1}$ for $\gamma-\mathrm{Fe}_{2} \mathrm{O}_{3}{ }^{38}$ The measured saturation magnetization of the NS89, NS205, and NS458 nanoparticles were $72.1,64.5$, and $77.4 \mathrm{~A} \cdot \mathrm{m}^{2} \cdot \mathrm{kg}^{-1}$, indicating that "X-ray-amorphous" $\mathrm{Fe}_{2} \mathrm{O}_{3}$ was present in the samples.

Micro-Raman spectroscopy was used to prove the existence of $\gamma-\mathrm{Fe}_{2} \mathrm{O}_{3}$ and $\alpha-\mathrm{Fe}_{2} \mathrm{O}_{3}$ in our samples by measurements of 

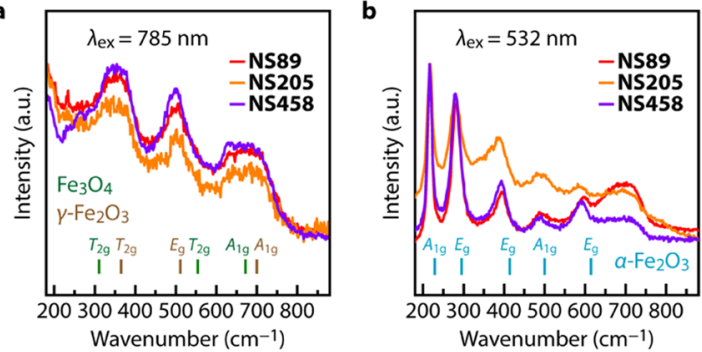

Figure 2. Micro-Raman spectra of $\mathrm{Fe}_{3} \mathrm{O}_{4}$ nanoparticles with diameters of $89 \pm 19 \mathrm{~nm}$ (NS89), $205 \pm 27 \mathrm{~nm}$ (NS205), and $458 \pm 137 \mathrm{~nm}$ (NS458) under excitation wavelengths $\left(\lambda_{\text {ex }}\right)$ of (a) 785 and (b) 532 nm. The Raman active modes in $\mathrm{Fe}_{3} \mathrm{O}_{4}, \gamma-\mathrm{Fe}_{2} \mathrm{O}_{3}$, and $\alpha-\mathrm{Fe}_{2} \mathrm{O}_{3}$ are given in the figures for comparison.

off-resonance $(785 \mathrm{~nm})$ and on-resonance $(532 \mathrm{~nm})$ excitations (Figure 2). Under on-resonance conditions, the excitation laser wavelength (corresponding to $2.3 \mathrm{eV}$ ) was matched to an electronic transition in hematite, thus enhancing the signal from this material. The off-resonance spectra were taken without resonance enhancement and no proximity to electronic transitions. The off-resonance spectra, taken with $785 \mathrm{~nm}$ excitation (Figure 2a), show three broad peaks, which can be attributed to a mixture of $\mathrm{Fe}_{3} \mathrm{O}_{4}\left(\mathrm{~T}_{2 \mathrm{~g}}, \mathrm{~T}_{2 \mathrm{~g}}\right.$, and $\left.\mathrm{A}_{1 \mathrm{~g}}\right)$ and maghemite, $\gamma-\mathrm{Fe}_{2} \mathrm{O}_{3}\left(\mathrm{~T}_{2 \mathrm{~g}}, \mathrm{E}_{\mathrm{g}}\right.$, and $\left.\mathrm{A}_{1 \mathrm{~g}}\right)$. ${ }^{39}$ In the on-resonance spectra, acquired with $532 \mathrm{~nm}$ excitation (Figure $2 \mathrm{~b}$ ), peaks at $218,281,393,492$, and $593 \mathrm{~nm}$ can be ascribed to the $A_{1 g}, E_{g}$, $\mathrm{E}_{\mathrm{g}}, \mathrm{A}_{1 \mathrm{~g}}$, and $\mathrm{E}_{\mathrm{g}}$ modes of hematite $\left(\alpha-\mathrm{Fe}_{2} \mathrm{O}_{3}\right){ }^{39}$ Thus, microRaman spectra indicate the presence of a surface $\mathrm{Fe}_{2} \mathrm{O}_{3}$ layer, mainly consisting of $\gamma-\mathrm{Fe}_{2} \mathrm{O}_{3}$, while $\alpha-\mathrm{Fe}_{2} \mathrm{O}_{3}$ was present in a small fraction in the surface layer and was detected only by onresonance excitation.

The $\mathrm{Fe}_{3} \mathrm{O}_{4}$ nanoparticles were dispersed in a water and poly(vinylpyrrolidone) (PVP) solution and encapsulated between two glass slides separated with a spacer of thickness around $80 \mu \mathrm{m}$. The volume fraction was $0.067 \%$, and hence the samples should be within the independent scattering region in the absence of clustering. ${ }^{40}$ The samples exhibited different colors (Figure S2 insets) from yellowish (NS89), and brownish
(NS205) to black (NS458). Since $\mathrm{Fe}_{3} \mathrm{O}_{4}$ is a black ferromagnetic iron oxide with both $\mathrm{Fe}(\mathrm{III})$ and $\mathrm{Fe}(\mathrm{II})$ valence states, ${ }^{1}$ a yellowish and brownish color might be due to a surface layer of $\mathrm{Fe}_{2} \mathrm{O}_{3}$. The total, diffuse, and regular/specular transmittance and reflectance were characterized by a spectrophotometer. Initially, the optical properties of the samples changed after about 20 days, probably due to particle aggregation or settling, as indicated by spectrophotometric data taken at different times after preparation (Figure S3). These changes were small for the NS89 sample and most significant for the NS458 sample. These effects may conceivably be connected to particle aggregation processes in the samples, which were pronounced for the $458 \mathrm{~nm} \mathrm{Fe}_{3} \mathrm{O}_{4}$ particles, as observed by optical microscopy (Figure S2). The optical data presented below were always measured when the optical properties were stable. Figure 3 shows spectrophotometric transmittance and reflectance data for $\mathrm{Fe}_{3} \mathrm{O}_{4}$ nanoparticles of three different sizes, dispersed in a water and PVP matrix.

2.2. Angle- and Wavelength-Resolved Light Scattering. A detailed wavelength- and angle-resolved scattering characterization of the $\mathrm{Fe}_{3} \mathrm{O}_{4}$ nanocomposite layers was carried out using an in-house scatterometer, which measures the forward and backward scattered intensity as a function of angle and wavelength (Section 4). First, the dependence on the azimuthal angle of the scattering intensity distribution was measured by a goniometer (Figure S4) using a $633 \mathrm{~nm} \mathrm{He}-\mathrm{Ne}$ laser. The results clearly show that scattering is axially symmetric and only depends on the polar angle, $\theta$. The scattering angle $\beta_{\mathrm{o}}$ is defined to be in the range of $0-90^{\circ}$, in both the forward and backward scattering hemispheres, while $\theta$ goes from 0 to $180^{\circ}$. Hence, $\beta_{\mathrm{o}}\left(0,90^{\circ}\right)$ corresponds to $\theta(0$, $\left.90^{\circ}\right)$ in the forward region and $\theta\left(180,90^{\circ}\right)$ in the backward region. The scattered intensity is presented as angle-resolved scattering (ARS), ${ }^{41}$ defined as

$$
\operatorname{ARS}(\beta)=\frac{S(\beta)}{P_{\mathrm{i}} \times \Omega}
$$
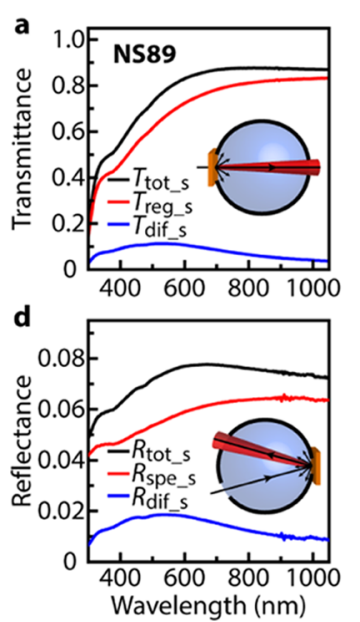

b
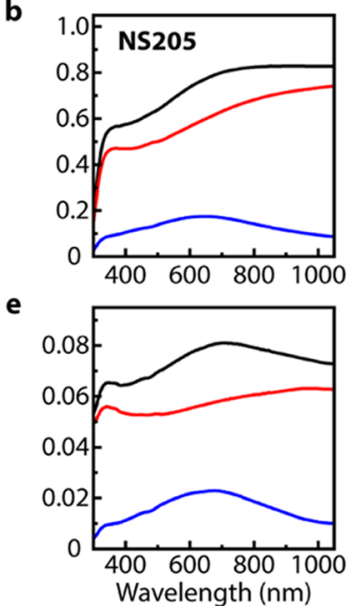

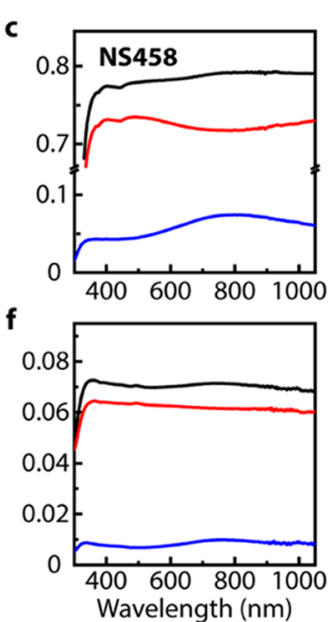

Figure 3. $(\mathrm{a}-\mathrm{c})$ Total, regular, and diffuse transmittance $\left(T_{\text {tot }}, T_{\text {reg }}\right.$, and $\left.T_{\text {dif }}\right)$ of the $\mathrm{Fe}_{3} \mathrm{O}_{4}$ NS89, NS205, and NS458 particles dispersed in water-PVP (subscript $s$ represents the stable state). The volume fraction was $0.067 \%$ and the thicknesses of the scattering layers were 72,76 , and $76 \mu \mathrm{m}$, respectively. (d-f) Total, specular, and diffuse reflectance $\left(R_{\text {tot }}, R_{\text {spe }}\right.$ and $\left.R_{\text {dif }}\right)$ of the same $\mathrm{Fe}_{3} \mathrm{O}_{4}$ samples. Insets are schematics of transmittance (a) and reflectance (d) measurements with an integrating sphere. Red cones denote regular transmitted and specular reflected lights and blue spheres represent the diffuse transmitted and reflected light. 


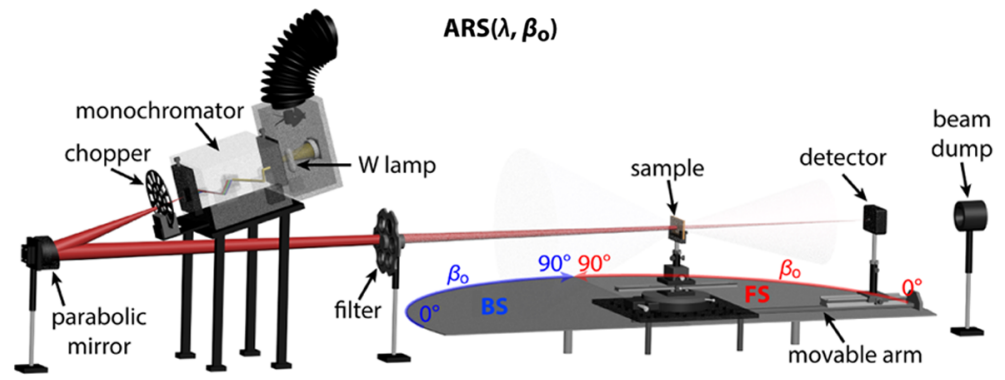

b
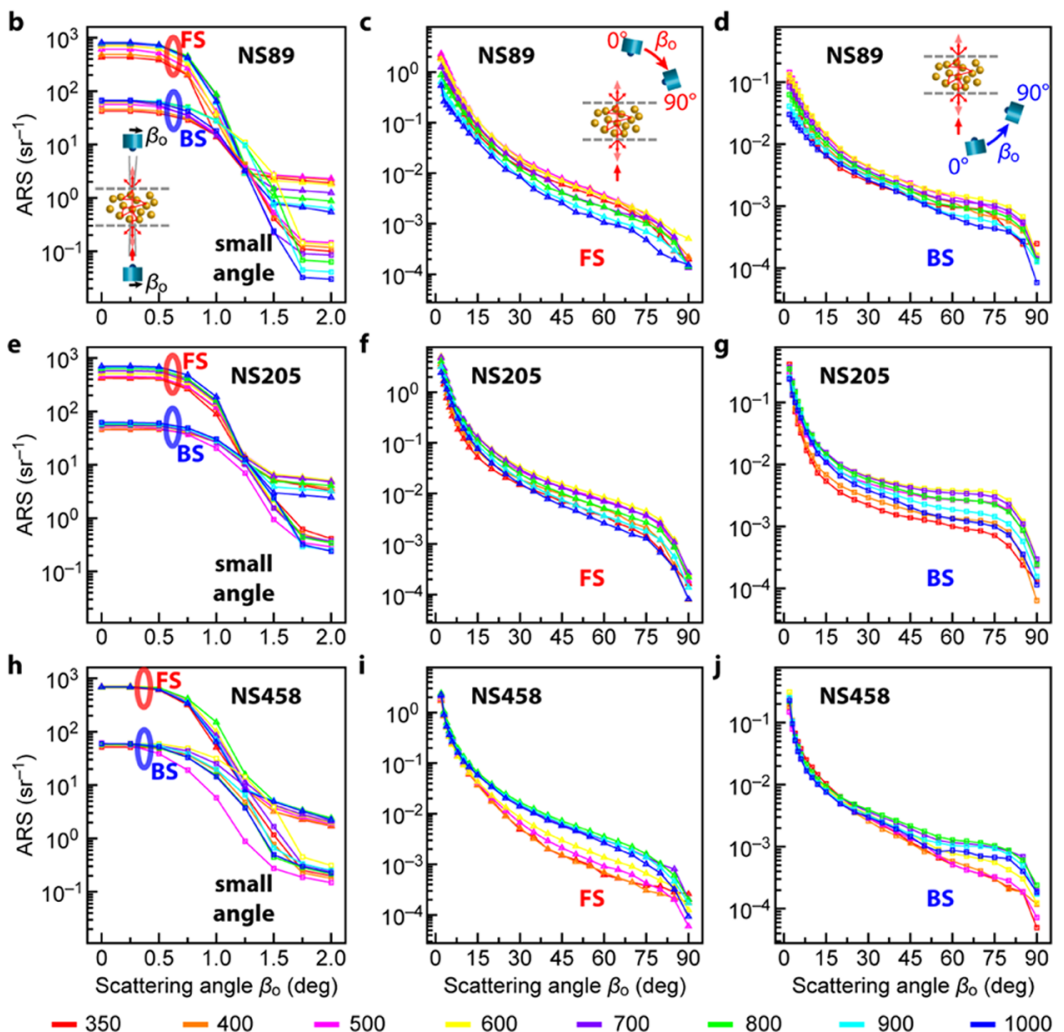

Figure 4. Schematic illustration of angle- and wavelength-resolved measurement using the in-plane scatterometer (a) in the forward and backward regions. Scattered intensity as a function of the scattering angle, at different wavelengths, for $\mathrm{Fe}_{3} \mathrm{O}_{4}$ nanocomposite samples with particle diameters of $89 \mathrm{~nm}(\mathrm{~b}-\mathrm{d}), 205 \mathrm{~nm}(\mathrm{e}-\mathrm{g})$, and $458 \mathrm{~nm}(\mathrm{~h}-\mathrm{j})$. The volume fraction was $0.067 \%$ and the thicknesses of the scattering layers were 72,76 , and $76 \mu \mathrm{m}$, respectively. The figures show the small-angle-transmitted intensity (forward scattering, FS) and small-angle-reflected intensity (backward scattering, BS) (b, e, h), the large-angle-transmitted intensity (forward scattering, FS) (c, f, i) and the large-angle-reflected intensity (backward scattering, BS) (d, g, j). The legends below denote eight different incident light wavelengths. Forward scattering (FS) and backward scattering (BS) are denoted by triangles $(\Delta)$ and squares $(\square)$, respectively.

where $S(\beta)$ is the measured radiant intensity from the sample, $P_{\mathrm{i}}$ is the light intensity incident on the sample, and $\Omega$ is the solid angle of the detector. Figure 4 shows angular profiles at small angles (left column), and in the whole angular range in the forward direction (middle column) and backward direction (right column) for the NS89, NS205, and NS458 $\mathrm{Fe}_{3} \mathrm{O}_{4}$ nanocomposite samples measured on the in-plane scatterometer (Figure 4a). The $\mathrm{Fe}_{3} \mathrm{O}_{4}$ samples show a significant preference for forward scattering. In weakly scattering samples, a significant amount of light is directly transmitted and part of it is reflected at the back interface. This reflected light experiences scattering by particles on its way back to the front interface. These effects give strong forward and backward peaks in the scattering pattern. ${ }^{36,37}$ At small angles $\left(\beta<2^{\circ}\right)$, the major part of the measured transmitted intensity originates from light that is neither absorbed nor scattered by the particles. A smaller part is due to light that is scattered and diffracted by the particles at small angles. It is seen that the directly transmitted light is $2-3$ orders of magnitude higher than the scattered light and dominates at angles below $2^{\circ}$. The reflected intensity in the small-angle region is due to the specular reflectance from the front and back glass-air interfaces as well as the backscattered light by the particles. For larger angles, $\beta>2^{\circ}$, the measured ARS purely originates from the scattering by the particles (diffuse scattering), and it is seen that this contribution monotonically decreases toward higher angles. The features of the ARS spectra are similar for all wavelengths in the interval of 350-1000 nm. A faster drop in intensity in the forward direction shows that the lightscattering pattern is more anisotropic in the forward and more isotropic in the backward hemisphere.

The measured angle-resolved scattering intensity can be integrated over the forward and backward hemispheres to obtain the diffuse and regular/specular transmittance and 

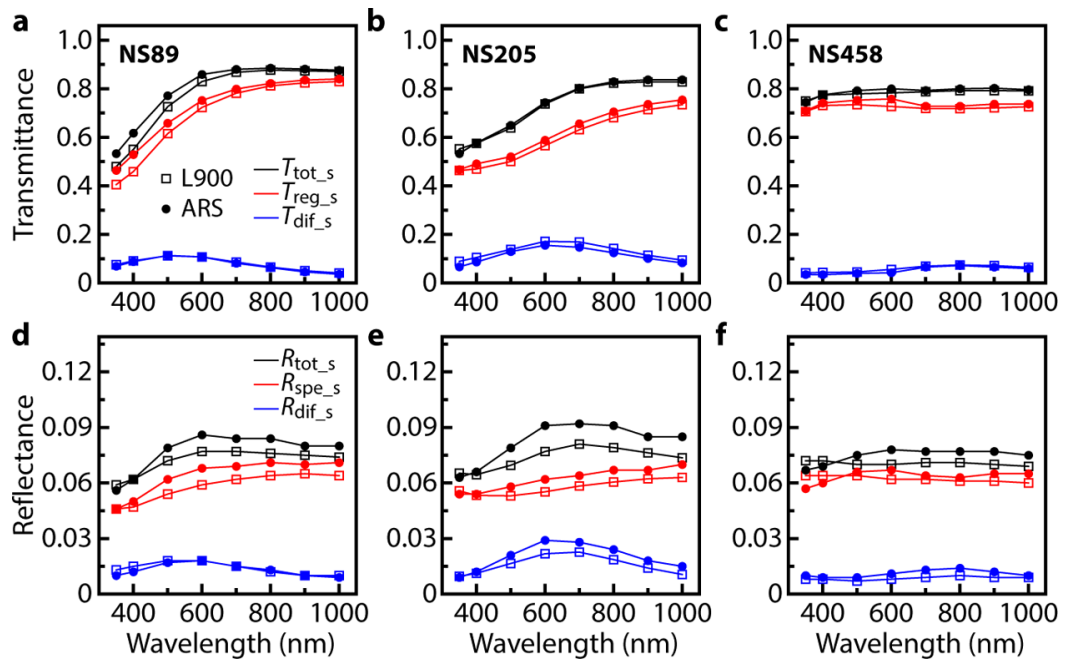

Figure 5. Comparison of integrated angle-resolved intensities (open squares) with spectrometric measurements (solid circles) of total, regular/ specular, and diffuse transmittance and reflectance for NS89 (a, d), NS205 (b, e), and NS458 (c, f) $\mathrm{Fe}_{3} \mathrm{O}_{4}$ nanocomposite samples. The volume fraction was $0.067 \%$ and the thicknesses of the scattering layers were 72,76 , and $76 \mu \mathrm{m}$, respectively.
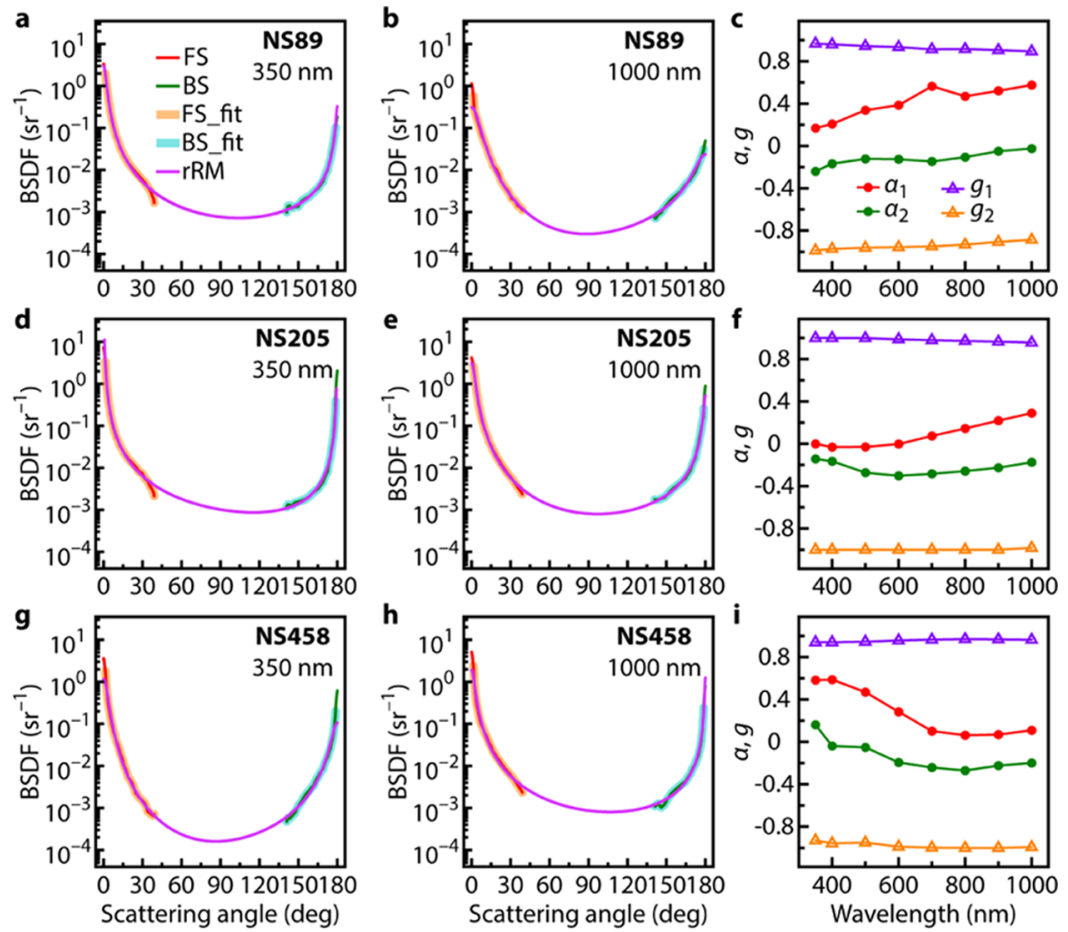

Figure 6. Scattering intensity distributions as a function of the polar angle inside the layer (red and green curves) for NS89, NS205, and NS458 $\mathrm{Fe}_{3} \mathrm{O}_{4}$ nanocomposite samples, and the resulting fits by the revised Reynolds-McCormick (rRM) scattering-phase function (violet curves) at wavelengths $350 \mathrm{~nm}$ (a, d, g; left column) and $1000 \mathrm{~nm}$ (b, e, h; middle column). The volume fraction was $0.067 \%$ and the thicknesses of the scattering layers were 72,76 , and $76 \mu \mathrm{m}$, respectively. The fitting parameters were $g_{1}, g_{2}, \alpha_{1}$, and $\alpha_{2}$ (c, f, i; right column). FS_fit and BS_fit denote the angular ranges used for the rRM fits in the forward and backward directions.

reflectance. We then compared the integrated $\operatorname{ARS}(\beta)$ with measured values of $T_{\mathrm{dif}}, R_{\mathrm{dif}}, T_{\text {reg, }}$ and $R_{\text {spe }}$ obtained from the spectrophotometer. In the case when the scattering is not dependent on the azimuthal angle, the integration was carried out according to ${ }^{41}$

$$
\begin{aligned}
T_{\text {dif }} & =\int_{\text {reg }}^{90^{\circ}} 2 \pi \operatorname{ARS}(\beta) \sin (\beta) \mathrm{d} \beta \\
R_{\text {dif }} & =\int_{\text {spe }}^{90^{\circ}} 2 \pi \operatorname{ARS}(\beta) \sin (\beta) \mathrm{d} \beta
\end{aligned}
$$

$$
\begin{aligned}
T_{\text {reg }}= & T\left(0^{\circ}\right)+\int_{0^{\circ}}^{2^{\circ}} 2 \pi \operatorname{ARS}_{\text {ext }}(\beta) \sin (\beta) \mathrm{d} \beta \\
& +\int_{2^{\circ}}^{\text {reg }} 2 \pi \operatorname{ARS}(\beta) \sin (\beta) \mathrm{d} \beta
\end{aligned}
$$

$$
\begin{aligned}
R_{\text {spe }}= & R\left(0^{\circ}\right)+\int_{0^{\circ}}^{2^{\circ}} 2 \pi \mathrm{ARS}_{\text {ext }}(\beta) \sin (\beta) \mathrm{d} \beta \\
& +\int_{2^{\circ}}^{\text {spe }} 2 \pi \operatorname{ARS}(\beta) \sin (\beta) \mathrm{d} \beta
\end{aligned}
$$



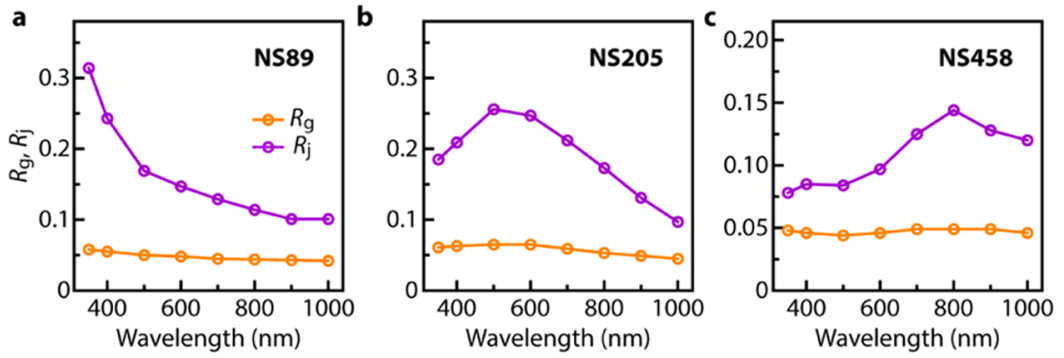

Figure 7. Front and back interface reflectances $R_{\mathrm{j}}$ and $R_{\mathrm{g}}$ from eqs 8 and 9, using measurements obtained by the ARS method (Figure 6) for (a) NS89, (b) NS205, and (c) NS458 $\mathrm{Fe}_{3} \mathrm{O}_{4}$ nanocomposite samples.

where reg and spe represent the upper limit of the angular ranges for $T_{\text {reg }}$ and $R_{\text {spe }}$, as determined by the geometry of the integrating sphere of the spectrophotometer, i.e., 4.6 and $7.4^{\circ}$, respectively. The symbol $\mathrm{ARS}_{\text {ext }}(\beta)$ denotes the extrapolation of the diffuse scattering intensity from 2 to $0^{\circ}$. $T\left(0^{\circ}\right)$ and $R\left(0^{\circ}\right)$ are the measured ARS transmittance and reflectance with the detector positioned at $0^{\circ}$. Figure 5 shows the integrated ARS results in comparison with the transmittance and reflectance spectra measured by the spectrophotometer. The results are in good agreement, proving the accuracy of the instruments and the measurement procedures.

2.3. Determination of the Interfacial ScatteringPhase Function. The objective of this work is to invert spectrophotometric and ARS data to calculate the backscattering and absorption coefficients of the composites. The ARS measurements are needed for determining the interface reflectances for light coming from the inner scattering layer. Calculating the interface reflectances requires knowledge of the scattering intensity distribution for all angles from 0 to $90^{\circ}$ within the scattering layer. It is only possible to measure the light-scattering intensity outside the sample and this quantity needs to be converted into the light intensity distributions inside the layer and close to the interfaces. The inside and outside angles are related by Snell's law and the intensity has to be modified by considering the interface reflectance obtained from Fresnel's equation (Section 4). The angle-dependent scattering intensity or the phase function inside the nanoparticle samples is displayed in Figure 6 (left and middle columns) at wavelengths of 350 and $1000 \mathrm{~nm}$. The red and green lines denote the inner scattering distributions in the forward and backward directions, respectively. Due to the refractive index difference from air to the sample, no information can be obtained for angles larger than the critical angle of total reflection for light propagating from the inside of the nanocomposite layer. A revised Reynolds-McCormick scattering-phase function was used to simulate the forward and backward branches (violet curve). The original form of the empirical Reynolds-McCormick (RM) approximation ${ }^{42}$ is

$$
\begin{aligned}
& f_{\mathrm{RM}}(\theta, \alpha, g) \\
& \quad=\frac{\alpha g\left(1-g^{2}\right)^{2 \alpha}}{\pi\left[1+g^{2}-2 g \cos (\theta)\right]^{\alpha+1}\left[(1+g)^{2 \alpha}-(1-g)^{2 \alpha}\right]}
\end{aligned}
$$

where the polar angle $\theta$ ranges from 0 to $180^{\circ}$ to cover both the forward and backward hemispheres, $g$ is the asymmetry factor, which can have values from 0 to \pm 1 , and $\alpha$ is a fitting parameter. We modified the RM model so that it can be fitted to both the forward and backward branches of strongly peaked distributions, in a manner similar to the two-term HenyeyGreenstein approximation ${ }^{43}$

$$
\begin{aligned}
& f_{\mathrm{rRM}}\left(\theta, \alpha_{1}, g_{1}, \alpha_{2}, g_{2}, \gamma\right) \\
& \quad=\gamma f_{\mathrm{RM}}\left(\alpha_{1}, g_{1}, \theta\right)+(1-\gamma) f_{\mathrm{RM}}\left(\alpha_{2}, g_{2}, \theta\right)
\end{aligned}
$$

where $\gamma$ is a new fitting parameter that describes the amplitude of the two terms. Figure 6 (right column) depicts the fitting parameters $g_{1}, g_{2}, \alpha_{1}$, and $\alpha_{2}$ of the revised ReynoldsMcCormick approximation in the wavelength range from 350 to $1000 \mathrm{~nm}$. It is seen that the experimental data can be very well fitted by eq 7 . The single-scattering-phase functions calculated by the Mie theory are shown in Figure S5. These calculations show a strongly forward peaked intensity, which becomes more dominant as the particle size increases. The differences between the scattering intensity distributions in Figure 6 and the single-scattering Mie calculations indicate that multiple scattering effects, as well as dependent scattering (particle-particle interference due to particle aggregation) may be important for our samples.

2.4. Extraction of Absorption and Scattering Coefficients. By knowing the scattering-phase function of the diffusely scattered light, internal interface reflectances can be obtained by considering both specular and diffuse light contributions of the radiation fluxes ${ }^{35}$

$$
R_{\mathrm{g}, \mathrm{j}}=\left(1-q_{0, \mathrm{~d}}\right) R_{\mathrm{c}}+q_{0, \mathrm{~d}} R_{\mathrm{df}, \mathrm{db}}
$$

where $R_{c}$ is the specular reflectance (Section 4) and $q_{0, \mathrm{~d}}$ denotes the diffuse light fractions in the film at the back (0) and front (d) interfaces ${ }^{35}$ (Section 4). The diffuse reflectances $R_{\mathrm{df}, \mathrm{db}}$ of light incident on the back and front interfaces, from the inside of the sample, can be calculated by integrating the scattering distribution functions in the forward hemisphere, $f_{t}(\beta)$, and in the backward hemisphere, $f_{r}(\beta)$, over all angles of incidence (Figure S6).

$$
R_{\mathrm{df}, \mathrm{db}}=\frac{\int_{0^{\circ}}^{90^{\circ}} f_{\mathrm{t}, \mathrm{r}}(\beta) r(\beta) \sin (2 \beta) \mathrm{d} \beta}{\int_{0^{\circ}}^{90^{\circ}} f_{\mathrm{t}, \mathrm{r}}(\beta) \sin (2 \beta) \mathrm{d} \beta}
$$

Figure 7 shows the front interface reflectance $R_{\mathrm{j}}$ and back interface reflectance $R_{\mathrm{g}}$ of the three samples. The parameter $R_{\mathrm{j}}$ has a higher value than $R_{\mathrm{g}}$, which shows that the backscattering is more diffuse than the forward one. This means that there is a larger portion of scattering at higher angles in the back hemisphere.

After these preliminaries, we now use the $\mathrm{KM}$ model to derive the backscattering and absorption coefficients $S$ and $K$. This involves the inversion of the Kubelka-Munk equations for total transmittance and reflectance, ${ }^{34,35}$ 

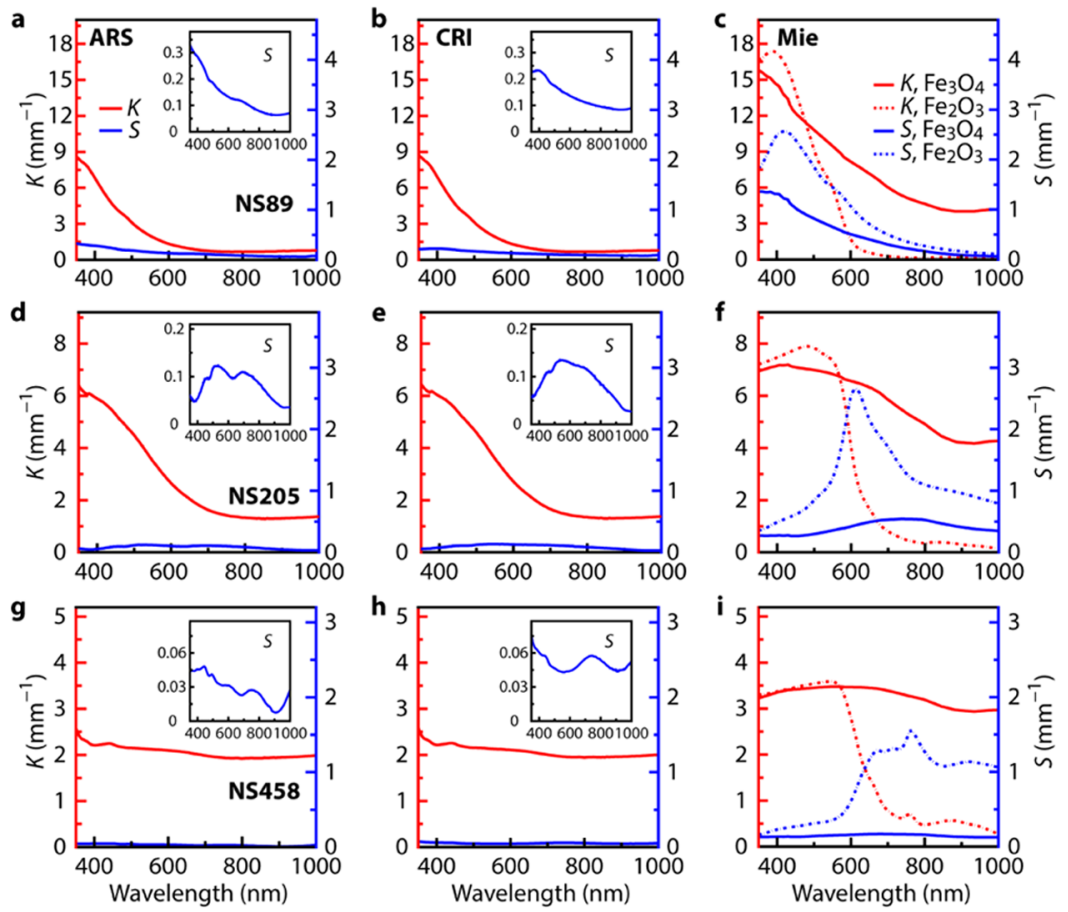

Figure 8. Fitted absorption coefficient $K$ (red) and backscattering coefficient $S$ (blue) by (a, d, g) ARS and (b, e, h) CRI methods for NS89, $\mathrm{NS} 205$, and NS458 $\mathrm{Fe}_{3} \mathrm{O}_{4}$ nanocomposite samples (insets show the backscattering coefficient $S$ on an expanded scale). The volume fraction was $0.067 \%$. (c, f, i) Simulated $K$ (red) and $S$ (blue) by the Mie theory for $\mathrm{Fe}_{3} \mathrm{O}_{4}$ (solid) and $\mathrm{Fe}_{2} \mathrm{O}_{3}$ (dotted) nanocomposites considering the particle size distributions of the NS89, NS205, and NS458 samples.

$$
R=R_{\mathrm{c}}+\frac{\left(1-R_{\mathrm{c}}\right)\left(1-R_{\mathrm{j}}\right) R_{\mathrm{KM}}}{1-R_{\mathrm{j}} R_{\mathrm{KM}}}
$$

and

$$
T=\frac{\left(1-R_{\mathrm{c}}\right) T_{\mathrm{KM}}}{1-R_{\mathrm{j}} R_{\mathrm{KM}}}
$$

where the optical properties of the diffuse light-scattering layer are described by ${ }^{34,35}$

$$
R_{\mathrm{KM}}=\frac{1-R_{\mathrm{g}}[a-b \operatorname{coth}(b S d)]}{a-R_{\mathrm{g}}+b \operatorname{coth}(b S d)}
$$

and

$$
T_{\mathrm{KM}}=\frac{b\left(1-R_{\mathrm{g}}\right)}{\left(a-R_{\mathrm{g}}\right) \sinh (b S d)+b \cosh (b S d)}
$$

In these equations, the albedo $a$ is equal to $(1+K / S)$ and $b$ is equal to $\left(a^{2}-1\right)^{0.5}$. When the reflectances $R_{\mathcal{c}} R_{\mathfrak{j}}$, and $R_{\mathrm{g}}$ have been determined, the only unknown parameters in these equations are the absorption coefficient $K$ and the backscattering coefficient $S$. Hence, these coefficients can be obtained from experimental reflectance and transmittance data and the fitting results are shown in Figure 8a,d,g. The inversion scheme that utilizes angle-resolved scattering measurements to determine $R_{\mathrm{j}}$ and $R_{\mathrm{g}}$ is denoted the ARS method. In our previous work, we also proposed an approximation denoted as the CRI method for the case when angle-resolved scattering data are not available. ${ }^{36}$ The CRI approximation ${ }^{36}$ assumes that the forward and backward scattering distributions are constant out to the critical angle of total reflection and that no light scattering happens at higher angles. Therefore, this approximation is suitable when the scattering patterns exhibit strong forward and backward scattering peaks. The CRI method is indeed a good approximation for the present type of samples, as shown before, ${ }^{36}$ since the scattering patterns are highly anisotropic (Figure 6) and exhibit large increases toward small forward and backward angles. Both the ARS and CRI methods converge and the calculated $T_{\text {tot }}$ and $R_{\text {tot }}$ reproduce the experimental results. The results from the CRI method are shown in Figure $8 \mathrm{~b}, \mathrm{e}, \mathrm{h}$ and are seen to be very similar to those of the ARS method. The coefficients $K$ and $S$ can also be compared to computations from the single-scattering Mie theory (Section 4), considering the experimental size distribution of the nanoparticles, and the results are shown in Figure 8c,f,i for both $\mathrm{Fe}_{3} \mathrm{O}_{4}$ and $\mathrm{Fe}_{2} \mathrm{O}_{3}$ particles. The reason for including both oxides is that an oxidized surface layer of $\mathrm{Fe}_{2} \mathrm{O}_{3}$ is present on the particles, as indicated by Raman spectra (Figure 2) and magnetic measurements (Figure S1). There is no quantitative agreement between our data and the Mie simulations, and our experimental scattering and absorption coefficients are always of lower magnitude than the Mie calculation results. However, qualitative similarities can be observed, most notably for the larger $\mathrm{Fe}_{3} \mathrm{O}_{4}$ particles, NS458, where $K$ exhibits a similar shape to that of the Mie simulation for $\mathrm{Fe}_{3} \mathrm{O}_{4}$. For the smaller particles, the absorption exhibits a shape that seems to be an intermediate between the $\mathrm{Fe}_{3} \mathrm{O}_{4}$ and $\mathrm{Fe}_{2} \mathrm{O}_{3}$ simulations. Probably, an oxidation layer is present on the small nanoparticles but has less influence on the larger particles. However, the backscattering is in all cases of much lower magnitude than in the Mie computations. The difference in the magnitude of $K$ is mainly due to particle deposition during stabilization of the samples since data for asprepared samples are much closer in magnitude to the Mie simulation (Figure S7). Discrepancies in the magnitude of $S$ are still present and the lower experimental than the computed 


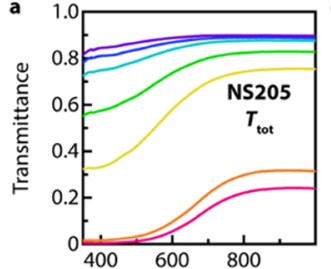

b

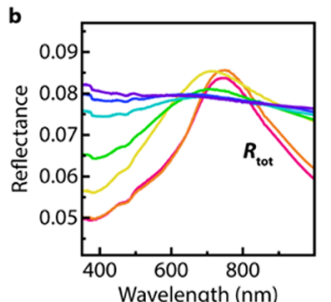

$-\begin{gathered}0.625 \\ \mathrm{mg} / \mathrm{mL}\end{gathered}-\begin{gathered}1.25 \\ \mathrm{mg} / \mathrm{mL}\end{gathered}$

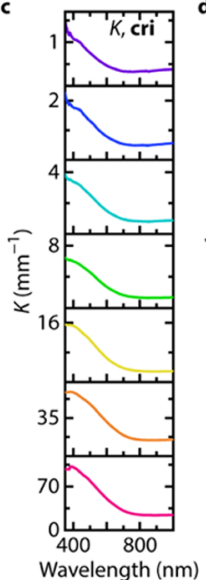

d
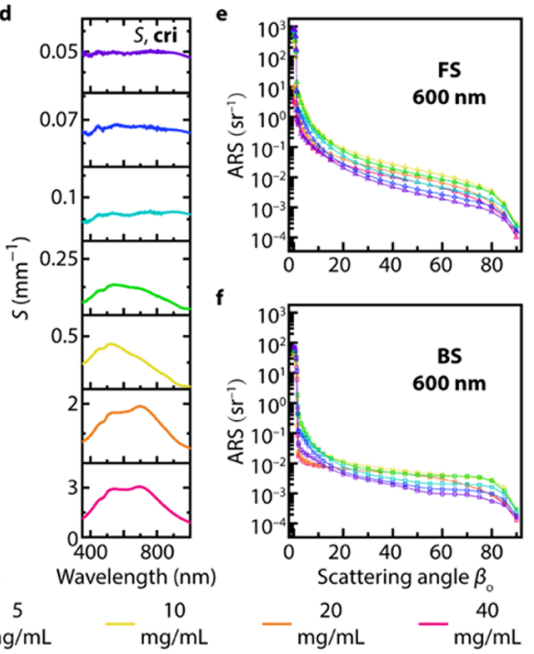

Figure 9. (a) $T_{\text {tot }}$ (b) $R_{\text {tot }}$ (c) absorption coefficient $K$, (d) backscattering coefficient $S$, and angle-resolved scattering at wavelength 600 nm in forward (e) and backward (f) directions for NS205 $\mathrm{Fe}_{3} \mathrm{O}_{4}$ nanoparticles in water and PVP prepared from dispersions in water with particle concentrations of $0.625,1.25,2.5,5,10,20$, and $40 \mathrm{mg} \cdot \mathrm{mL}^{-1}$.

scattering coefficient is probably an indication of dependent scattering in our large clusters of closely approaching particles (Figure S2). It should, however, be noted that ARS measurements were always carried out on stabilized samples and therefore the inversion results become more uncertain for the as-prepared samples.

2.5. Influence of Thickness and Concentration on $S$ and $K$ Determination. We now report results obtained from spectrophotometric measurements on a larger set of $\mathrm{Fe}_{3} \mathrm{O}_{4}$ nanoparticle samples with various particle concentrations and thicknesses. In this case, we use the CRI approach to calculate the backscattering and absorption coefficients of the samples since angle-resolved measurements were only carried out at $600 \mathrm{~nm}$ for each sample. The total transmittance and reflectance spectra of $\mathrm{Fe}_{3} \mathrm{O}_{4}$ NS205 samples prepared from nanoparticles in water with concentrations of $0.625,1.25,2.5$, $5,10,20$, and $40 \mathrm{mg} \cdot \mathrm{mL}^{-1}$, corresponding to the volume fractions of $0.008,0.017,0.034,0.067,0.134,0.268$, and $0.536 \%$ in water + PVP are displayed in Figure 9 together with the $S$ and $K$ results in a stable state. Figure $S 8$ shows corresponding results from measurements in the as-prepared state. It is clearly seen that both $S$ and $K$ increase with increasing concentration, as could be expected. Forward and backward angular scattering at a wavelength of $600 \mathrm{~nm}$ are depicted in Figure 9e,f, indicating that the $10 \mathrm{mg} \cdot \mathrm{mL}^{-1}$ sample exhibits strong scattering in both directions and a further increase of the concentration lowers the scattering intensity.

We also studied the thickness dependence of the transmittance, reflectance, and the $S$ and $K$ coefficients for the 2.5 $\mathrm{mg} \cdot \mathrm{mL}^{-1}$ (Figure S9 for the stable state and Figure S10 for the as-prepared state) and $10 \mathrm{mg} \cdot \mathrm{mL}^{-1}$ (Figure S11 for the stable state and Figure $\mathrm{S} 12$ for the as-prepared state) samples. The absorption coefficients show qualitatively similar shapes for samples of different thicknesses, while the shape of backscattering coefficient spectra for different samples vary, which might be due to multiple scattering and aggregation effects.

\section{CONCLUSIONS}

In this paper, $\mathrm{Fe}_{3} \mathrm{O}_{4}$ nanospheres with different sizes were synthesized and encapsulated into a polymer matrix, thereby forming nanoparticle composite layers. The optical trans- mittance and reflectance spectra, together with angle-resolved light-scattering spectra were investigated for these composite layers. The backscattering $(S)$ and absorption $(K)$ coefficient spectra were obtained by inversion of the Kubelka-Munk twoflux radiative transfer theory using spectrophotometric as well as angle-dependent measurements. The absorption of large $458 \mathrm{~nm}$ nanoparticles in the matrix shows qualitative agreement with single-scattering Mie calculations. Major discrepancies occur for smaller particles and these are due to the formation of $\mathrm{a} \mathrm{Fe}_{2} \mathrm{O}_{3}$ surface layer on the particles, as well as dependent scattering in clusters of particles. Concentration and thickness dependence were further studied using only transmittance and reflectance spectra and the approximate CRI method. The results show that $S$ is more sensitive than $K$ to thickness and concentration. We infer that multiple and dependent scatterings by aggregation influence both the shape and intensity of scattering spectra but only to a smaller extent the intensity of absorption.

\section{EXPERIMENTAL AND COMPUTATIONAL METHODS}

4.1. Material Synthesis and Characterization. All chemicals were obtained from Merck, Sigma-Aldrich, or VWR, and used without further purification. A bottom-up approach was used to fabricate all of the nanoparticle samples. This method allows for a good control over sizes and shapes of the nanostructure. In the fabrication of $\mathrm{Fe}_{3} \mathrm{O}_{4}$ NS89, iron(III) chloride hexahydrate $\left(\mathrm{FeCl}_{3} \cdot 6 \mathrm{H}_{2} \mathrm{O}, 0.05 \mathrm{~g}\right)$ and sodium acetate trihydrate $\left(\mathrm{NaAc} \cdot 3 \mathrm{H}_{2} \mathrm{O}, 0.15 \mathrm{~g}\right)$ were dissolved in 20 $\mathrm{mL}$ of ethylene glycol under ultrasonic stirring treatment. The obtained homogeneous yellow mixture was transferred to a $50 \mathrm{~mL}$ Teflon-lined stainless-steel autoclave and then heated to $200{ }^{\circ} \mathrm{C}$ for 4 h. After the reaction, the magnetic nanoparticles were collected by a magnet. They were suspended in $20 \mathrm{~mL}$ of ethylene glycol containing $\mathrm{FeCl}_{3} \cdot 6 \mathrm{H}_{2} \mathrm{O}(0.1 \mathrm{~g})$, poly(vinylpyrrolidone) (PVP, MW: 40000, $4 \mathrm{~g}$ ), $\mathrm{NaAc} \cdot 3 \mathrm{H}_{2} \mathrm{O}(0.3 \mathrm{~g})$, and $1 \mathrm{~mL}$ of poly(ethylene glycol) (PEG, MW: 300) under ultrasonic stirring treatment. The mixture was again heated to $200{ }^{\circ} \mathrm{C}$ for $4 \mathrm{~h}$ in an autoclave. To obtain $\mathrm{Fe}_{3} \mathrm{O}_{4} \mathrm{NS} 89$, the prepared solution was centrifuged at $1700 \mathrm{rpm}$ for $1 \mathrm{~min}$. $\mathrm{Fe}_{3} \mathrm{O}_{4} \mathrm{NSs}$ were collected from the supernatant by a magnet and washed with 50 $\mathrm{mL}$ of distilled water. For $\mathrm{Fe}_{3} \mathrm{O}_{4} \mathrm{NS} 205, \mathrm{FeCl}_{3} \cdot 6 \mathrm{H}_{2} \mathrm{O}(0.1 \mathrm{~g})$, PVP (MW: $40000,4 \mathrm{~g}), \mathrm{NaAc} \cdot 3 \mathrm{H}_{2} \mathrm{O}(0.3 \mathrm{~g})$, and $1 \mathrm{~mL}$ of PEG (MW: 300) were dissolved in $20 \mathrm{~mL}$ of ethylene glycol under ultrasonic stirring. The yellowish mixture was then moved to a $50 \mathrm{~mL}$ autoclave, 
which was heated to $200{ }^{\circ} \mathrm{C}$ for $4 \mathrm{~h}$. We collected the $\mathrm{Fe}_{3} \mathrm{O}_{4}$ nanoparticles using a magnet, followed by a purification process using a centrifuge at $1700 \mathrm{rpm}$ for $20 \mathrm{~min}$. The precipitated nanoparticles were washed with $50 \mathrm{~mL}$ of distilled water. For $\mathrm{Fe}_{3} \mathrm{O}_{4}$ NS458, $\mathrm{FeCl}_{3} \cdot 6 \mathrm{H}_{2} \mathrm{O}$ (0.8105 g), PVP (MW: $40000,2 \mathrm{~g}$ ), and NaAc. $3 \mathrm{H}_{2} \mathrm{O}(1.225 \mathrm{~g})$ were dissolved in $25 \mathrm{~mL}$ of ethylene glycol under ultrasonic stirring treatment. The homogeneous yellowish mixture was moved to a $50 \mathrm{~mL}$ autoclave, which was heated to $190{ }^{\circ} \mathrm{C}$ for 12 h. The $\mathrm{Fe}_{3} \mathrm{O}_{4} \mathrm{NSs}$ were purified by centrifugation at $1600 \mathrm{rpm}$ for 1 min. We washed the precipitate with $10 \mathrm{~mL}$ of distilled water. The centrifugation and washing processes for NS89, NS205, and NS458 were repeated five times. Subsequently, all of the samples were prepared by drying in an oven overnight at $80{ }^{\circ} \mathrm{C}$.

For the experiments reported in Sections 2.1 to 2.4, $\mathrm{Fe}_{3} \mathrm{O}_{4} \mathrm{NSs}$ particles were added to water at a concentration of $5 \mathrm{mg} \cdot \mathrm{mL}^{-1}$, equivalent to a volume fraction of $0.1 \%$, and corresponding to a particle concentration of $2.39 \times 10^{11} \mathrm{~mL}^{-1}$. Subsequently, we added PVP into the NSs and water solution. The mass ratio between PVP to water was 0.8 . The NS, water, and PVP mixture was shaken vigorously in a vortex mixer until the PVP powder was totally dissolved. The gellike solution was deposited on a glass slide, and subsequently, a spacer with a thickness of $\sim 80 \mu \mathrm{m}$ and another glass slide were added on top. The resulting sample with particles in water and PVP was sealed with glue and measurements were performed after 20 days when the sample was stable and its transmittance and reflectance remained unchanged. The sample preparation for the experiments in Section 2.5 was carried out in the same way but using different concentrations of $\mathrm{Fe}_{3} \mathrm{O}_{4} \mathrm{NSs}$ in water and different spacer thicknesses.

Scanning electron microscopy was performed on a Zeiss (LEO) 1530 FEG microscope. The acceleration voltage was $5 \mathrm{keV}$. X-ray diffraction patterns were measured by a SIEMENS D5000 diffractometer with $\mathrm{Cu} K \alpha(\lambda=1.54 \AA)$ radiation. The scanning speed was $0.1^{\circ} \cdot \mathrm{s}^{-1}$. Magnetization curves were measured by a vibrating sample magnetometer (VSM) from LakeShore at room temperature. In these measurements, the $\mathrm{Fe}_{3} \mathrm{O}_{4}$ samples were in a powder form and magnetization curves were normalized by weight. The error of the saturation magnetization was estimated to be $\pm 1 \mathrm{~A}$. $\mathrm{m}^{2} \cdot \mathrm{kg}^{-1}$, mainly coming from the weighing error.

Micro-Raman spectra were collected by two confocal Raman microscopes excited by two wavelengths (inVia Reflex, Renishaw, 532 $\mathrm{nm}, 2400$ lines per mm grating, and inVia Qontor, Renishaw, $785 \mathrm{~nm}$, 1200 lines per $\mathrm{mm}$ grating). The excitation laser intensities were 0.9 and $0.84 \mathrm{~mW}$ for 785 and $532 \mathrm{~nm}$, respectively. The laser beam was focused on the samples through a Leica $50 \times$ objective onto a $2 \mu \mathrm{m}$ spot. Measurements using different intensities, including low ones, were routinely conducted to ensure that the micro-Raman spectra did not change with light intensity; hence, the effects from laser-induced heating can be neglected. The results were obtained from an average of 50 scans with $10 \mathrm{~s}$ duration for each scan. Different places on the same samples were measured to eliminate possible false signals.

4.2. Optical Characterization. A Perkin-Elmer Lambda 900 UV-vis-NIR spectrophotometer equipped with a $150 \mathrm{~mm}$ Spectralon-coated integrating sphere was used to measure the total and diffuse reflectance and transmittance spectra in the wavelength range of $300-1100 \mathrm{~nm}$. A photomultiplier was used in the UV/vis range $(\lambda<900 \mathrm{~nm})$ and a lead sulfide $(\mathrm{PbS})$ detector was used further out in the NIR range. A scan speed of $250 \mathrm{~nm} \cdot \mathrm{min}^{-1}$, and a slit width of $3 \mathrm{~nm}$ in the visible and $2 \mathrm{~nm}$ in the NIR region were used. Reflectance measurements were taken at an angle of incidence of $8^{\circ}$ using a Spectralon plate, with known wavelength-dependent reflectance, as a reference. Total transmittance $\left(T_{\text {tot }}\right)$ is the sum of regular transmittance $\left(T_{\text {reg }}\right)$ and diffuse transmittance $\left(T_{\text {dif }}\right)$, while total reflectance $\left(R_{\text {tot }}\right)$ is the sum of specular reflectance $\left(R_{\text {spe }}\right)$ and diffuse reflectance $\left(R_{\mathrm{dif}}\right)$. Because of the geometry of the integrating sphere, the regular transmittance is defined as the directly transmitted light within an angle of $\pm 4.6^{\circ}$ from the normal, while the specular reflectance is defined as the directly reflected light within $\pm 7.4^{\circ}$. The scattered light at angles larger than $4.6^{\circ}$ in transmittance and $7.4^{\circ}$ in reflectance is taken to contribute to the diffuse transmittance and diffuse reflectance, respectively. To obtain $T_{\text {tot }} T_{\text {reg, }}$ and $T_{\text {dif }}$ as well as
$R_{\text {tot }}, R_{\text {spe }}$, and $R_{\text {dif }}$ the measured transmittance and reflectance signals must be corrected for the wavelength-dependent reflectance of the Spectralon reference, as well as for the difference between the reflectance of the reference plate and the coating on the integrating sphere and for geometrical factors such as port losses. The latter factors were determined by calibration with an absolute optical instrument. ${ }^{44}$ The correction procedure is described in detail in ref 36 and will not be repeated here.

The "in-plane" spectral- and angular-resolved spectrometer consisted of a $250 \mathrm{~W}$ tungsten-halogen lamp with a DC power supply, two gratings with blaze wavelengths of 0.75 and $2.0 \mu \mathrm{m}$, a monochromator, and an off-axis parabolic aluminum mirror. A silicon diode detector with a port dimension of $6.4 \times 6.4 \mathrm{~mm}^{2}$ and a good spectral response in the wavelength region from 300 to $1100 \mathrm{~nm}$ was used together with a lock-in amplifier (EG\&G model 7260). The sample was centrally placed and the silicon detector could be positioned at any angle from 0 to $180^{\circ}$. The illuminated spot was much smaller than the port of the Si detector and spanned an angle of $\pm 1^{\circ}$. A beam dump (Thorlabs) was applied to absorb the direct unscattered beam. To increase the dynamic range of the measurement setup, different neutral density (ND) filters (Thorlabs) were used at different wavelengths to attenuate the incoming light beam during measurements at small scattering angles. ${ }^{36}$ The intensity from the light source at the sample position was calculated from the intensity $S_{i}\left(0^{\circ}\right)$, measured using an ND filter in front of the detector, which was then divided by the filter transmittance at each wavelength. The transmittance and reflectance were measured as a function of angle at each chosen wavelength. An angle of incidence of $10^{\circ}$ was used for reflectance measurements to avoid shadowing of the incident light by the detector when measuring specular and near-specular reflected intensity. We present the measured transmitted and reflected intensity as angle-resolved scattering (ARS) by normalizing with the incident light intensity $P_{\mathrm{i}}$ at the sample position and the solid angle $\Omega$ of the detector $^{41}$

$$
\operatorname{ARS}(\beta)=\frac{S(\beta)}{P_{\mathrm{i}} \times \Omega}
$$

where $S(\beta)$ is the measured intensity from the sample as a function of the scattering angle. In the small-angle region, i.e., at angles smaller than $1^{\circ}$, the measurements were taken using ND filters, as described above, because of the high light intensity at these small angles. In these cases, $P_{\mathrm{i}}$ is equal to $S_{\mathrm{i}}\left(0^{\circ}\right)$. Furthermore, $\Omega=A / R^{2}$, where $A$ is the area of the detector port and $R$ is the distance from the sample to the detector, i.e., $200 \mathrm{~mm}$. The solid angle $\Omega$ was equal to $6.4^{2} / 200^{2}=$ $0.001024 \mathrm{sr}^{-1}$.

Since the scattering from our samples is quite low, especially at large angles, the accuracy of the instrument as well as the level of stray light must be ascertained. To this end, we have carried out background signal measurements for angles between 2 and $178^{\circ}$, i.e., away from the direct beam. At a wavelength of $600 \mathrm{~nm}$, we performed ARS measurements by (1) blocking the light close to the parabolic mirror and (2) by measuring light intensity as a function of angle without any sample ("blank" measurement) (Figure S13a). When the light is blocked, the signal is at a constant value, meaning that we have a good "dark" environment. The ARS values are around $10^{-5}\left(\mathrm{sr}^{-1}\right)$, probably from the electric noise of the instrument and quantify the limit of the detector. The blank measurement gives higher values, which are a measure of the stray light in the instrument setup, but these values were always much lower than the signals measured from the samples.

We have also considered the possibility of correcting the ARS intensity by subtracting the signals measured on a glass slide (Figure S13) to remove the background noise from stray light as well as the influence from scattering in the glass slides enclosing the sample. The signals measured from glass were close to the blank measurement at large scattering angles. Excess scattering above that of the blank was observed at relatively small angles especially in the backward direction, and this originates probably from scattering by surface roughness. The wavelength dependence of this background scattering 
was very small (Figure S13b). Subtracting the glass ARS intensity from the measured one for our samples led to insignificant differences (Figure S14).

The dependence of the scattering intensity distribution on the azimuthal angle was measured by a goniometer, ${ }^{45}$ using a red $\mathrm{He}-\mathrm{Ne}$ laser (wavelength $\lambda=633 \mathrm{~nm}$ ) as the light source. A positioncontrolled detector was mounted on a movable arm controlled by a step motor, thereby allowing the detector to scan in a hemisphere with a radius of $45 \mathrm{~cm}$.

4.3. Conversion of Outer Measured ARS into the Bidirectional Scattering Distribution Function (BSDF) Inside the Scattering Layer. We now describe a method to obtain the angular light intensity inside the scattering layer from ARS measurements. The outer and inner angles and the corresponding intensities need to be correlated by Snell's law and Fresnel's equations. ${ }^{41}$ First, Snell's law is used to derive the angle of propagation of light inside the layer $\left(\beta_{\mathrm{i}}\right)$ from the corresponding angle outside the layer, i.e., the scattering angle $\left(\beta_{\mathrm{o}}\right)$. To achieve this, we take into account the refractive index difference of the host (glass/(water + PVP)/glass), $n_{\mathfrak{j}}$, to that of the outside medium (i.e., air), $n_{\mathrm{o}}$.

$$
\beta_{\mathrm{i}}=\arcsin \left(\frac{\sin \left(\beta_{o}\right)}{\frac{n_{\mathrm{i}}}{n_{\mathrm{o}}}}\right)
$$

Second, the measured ARS is used to obtain the BSDF (denoted $f$ ) by dividing by the cosine correction factor, $\cos (\beta)$, to correct the surface area at the viewing angle. The phase function represents the angular distribution of the scattered radiation inside a material and is hence similar to the BSDF. We must also correct for the Fresnel reflectance of light passing from the sample to air, $r\left(\beta_{\mathrm{i}}\right)$, and thereby obtain the relation

$$
f\left(\beta_{\mathrm{i}}\right)=\frac{\operatorname{ARS}\left(\beta_{\mathrm{i}}\right)}{\left[1-r\left(\beta_{\mathrm{i}}\right)\right] \cos \left(\beta_{\mathrm{i}}\right)}
$$

The coefficient $r\left(\beta_{\mathrm{i}}\right)$ was obtained from the Fresnel equations for unpolarized light coming from inside of the light-scattering layer and incident at an angle $\beta=\beta_{\mathrm{i}}$ on the interface to air. ${ }^{46}$

$$
\begin{aligned}
r(\beta)= & \frac{1}{2}\left[r_{\mathrm{s}}(\beta)+r_{\mathrm{p}}(\beta)\right] \\
= & \frac{1}{2}\left(\left|\frac{n \cos (\beta)-n_{\text {air }} \sqrt{1-\left[\frac{n}{n_{\text {air }}} \sin (\beta)\right]^{2}}}{n \cos (\beta)+n_{\text {air }} \sqrt{1-\left[\frac{n}{n_{\text {air }}} \sin (\beta)\right]^{2}}}\right|^{2}+\right. \\
& \left|\frac{n \sqrt{1-\left[\frac{n}{n_{\text {air }}} \sin (\beta)\right]^{2}}-n_{\text {air }} \cos (\beta)}{n \sqrt{1-\left[\frac{n}{n_{\text {air }}} \sin (\beta)\right]^{2}}+n_{\text {air }} \cos (\beta)}\right|^{2}
\end{aligned}
$$

where the subindices in $r_{\mathrm{s}, \mathrm{p}}(\beta)$ denote s- and p-polarized light, $n$ is the refractive index of the sample, and $n_{\text {air }}$ is the refractive index of air, which is equal to unity.

4.4. Determination of Optical Parameters. In eq $8, q_{0}$ and $q_{\mathrm{d}}$ are the diffuse light fractions in the scattering layer close to the back and front interfaces, respectively

$$
\begin{aligned}
& q_{0}=\frac{I(0)-I_{c}(0)}{I(0)} \\
& q_{d}=\frac{J(d)-J_{c}(d)}{J(d)}
\end{aligned}
$$

where $I$ and $J$ are the intensities traveling toward the back and front interfaces. The coordinates $d$ and 0 denote the front and back interfaces and, in addition, the subscript $c$ represents collimated light.
According to Barrios et al. ${ }^{35} q_{0, \mathrm{~d}}$ can be obtained from transmittance and reflectance spectra by the following equations.

$$
\begin{aligned}
& q_{0}=\frac{T_{\text {dif }}\left(1-R_{\mathrm{c}}\right)}{T_{\text {tot }}\left(1-R_{\mathrm{c}}\right)-T_{\mathrm{reg}}\left(R_{\mathrm{df}}-R_{\mathrm{c}}\right)} \\
& q_{\mathrm{d}}=\frac{R_{\mathrm{dif}}\left(1-R_{\mathrm{c}}\right)}{R_{\mathrm{tot}}\left(1-R_{\mathrm{c}}\right)-R_{\mathrm{spe}}\left(R_{\mathrm{db}}-R_{\mathrm{c}}\right)+R_{\mathrm{c}} R_{\mathrm{db}}-R_{\mathrm{c}}}
\end{aligned}
$$

From the obtained $q_{0}$ and $q_{\mathrm{d}}$, together with $R_{\mathrm{df}}$ and $R_{\mathrm{db}}$, as calculated from eq 9 , we are able to determine the total back and front interface reflectances $R_{\mathrm{g}}$ and $R_{\mathrm{j}}(\mathrm{eq} 8)$.The imaginary $(k)$ and real $(n)$ refractive indices of the matrix, together with the reflectance at the air and glass interface $\left(R_{c}\right)$ were calculated from measurements of $R_{\text {spe }}$ and $T_{\text {reg }}{ }^{47}$ for a glass/(water + PVP)/glass sample,

$$
\begin{aligned}
R_{\mathrm{c}}= & \frac{\left(T_{\mathrm{reg}}^{2}+2\right)-\left(R_{\mathrm{spe}}-1\right)^{2}}{2\left(2-R_{\mathrm{spe}}\right)} \\
& -\sqrt{\left|\frac{\left(T_{\mathrm{reg}}^{2}+2\right)-\left(R_{\mathrm{spe}}-1\right)^{2}}{2\left(2-R_{\mathrm{spe}}\right)}\right|^{2}-\frac{R_{\mathrm{spe}}}{2-R_{\mathrm{spe}}}} \\
k= & -\frac{\lambda}{4 \pi d} \operatorname{In}\left(\frac{R_{\mathrm{spe}}-R_{\mathrm{c}}}{R_{\mathrm{spe}} R_{\mathrm{c}}}\right) \\
n= & \frac{1+R_{\mathrm{c}}}{1-R_{\mathrm{c}}}\left[1+\sqrt{1-\left|\frac{1-R_{\mathrm{c}}}{1+R_{\mathrm{c}}}\right|^{2}\left(1+k^{2}\right)}\right]
\end{aligned}
$$

The extinction coefficient of the water + PVP matrix was assumed to be equal to $k$ of the glass/(water + PVP)/glass sample. The critical angle $\theta_{c}$ was determined from $n$ (real part of the refractive index) of a single glass slide as follows

$$
\theta_{\mathrm{c}}=\arcsin \left(\frac{n_{\text {air }}}{n}\right)=\arcsin \left(\frac{1}{n}\right)
$$

4.5. Calculations by the Mie Theory. The optical response of particles with a spherical shape can be calculated from the analytic expressions of the Mie theory. The Mie code was adapted from Bohren and Huffman's book. ${ }^{48}$ The real and imaginary refractive index data of $\mathrm{Fe}_{3} \mathrm{O}_{4}$ and $\mathrm{Fe}_{2} \mathrm{O}_{3}$ were adapted from Schlegel ${ }^{49}$ and Querry. ${ }^{50}$ The absorption and backscattering cross sections were computed by the Mie theory and converted to absorption and backscattering coefficients by (assuming independent scattering)

$$
\begin{gathered}
S=\frac{C_{\mathrm{bsca}}}{V_{\mathrm{f}}}=\frac{f C_{\mathrm{bsca}}}{\left(\frac{4}{3} \pi a^{3}\right)} \\
K=\frac{C_{\mathrm{abs}}}{V_{\mathrm{f}}}=\frac{f C_{\mathrm{abs}}}{\left(\frac{4}{3} \pi a^{3}\right)}
\end{gathered}
$$

where $a$ is the radius of the nanospheres and $f$ is the volume fraction. The scattering-phase functions for the iron-oxide nanospheres considering the size distribution measured from SEM images were calculated by an online program Mieplot v4.6. ${ }^{51}$

\section{ASSOCIATED CONTENT}

\section{Supporting Information}

The Supporting Information is available free of charge at https://pubs.acs.org/doi/10.1021/acsanm.0c02309.

The description of the magnetization curves; optical microscopy images of the samples; transmittance and reflectance spectra; measurements by the out-of-plane goniometer; scattering-phase functions from the Mie theory; background signal measurements; diffuse 
reflectance, as well as absorption and backscattering coefficients for iron-oxide nanocomposite samples with different particle concentrations and thicknesses (PDF)

\section{AUTHOR INFORMATION}

\section{Corresponding Authors}

Changgang Xu - Department of Materials Science and Engineering, The Angström Laboratory, Uppsala University, SE-75103 Uppsala, Sweden; School of Materials Science and Engineering, Xi'an University of Science and Technology, Xi'an 710054, China; Email: changgangxu@xust.edu.cn

Gunnar A. Niklasson - Department of Materials Science and Engineering, The Ångström Laboratory, Uppsala University, SE-75103 Uppsala, Sweden; 이이. orcid.org/0000-0002-82795163; Email: Gunnar.Niklasson@angstrom.uu.se

\section{Authors}

Junxin Wang - Department of Materials Science and Engineering, The Angström Laboratory, Uppsala University, SE-75103 Uppsala, Sweden

Hui Xiong - Department of Materials Science and Engineering, The Ångström Laboratory, Uppsala University, SE-75103 Uppsala, Sweden; School of Mathematics and Physics, Hubei Polytechnic University, Huangshi 435003, Hubei, China

Yuanyuan Han - Department of Materials Science and Engineering, The Ångström Laboratory, Uppsala University, SE-75103 Uppsala, Sweden

Annica M. Nilsson - Department of Materials Science and Engineering, The Ångström Laboratory and Department of Civil and Industrial Engineering, The Angström Laboratory, Uppsala University, SE-75103 Uppsala, Sweden

Mattias Strömberg - Department of Materials Science and Engineering, The Angström Laboratory, Uppsala University, SE-75103 Uppsala, Sweden; 이이.org/0000-0003-06483130

Tomas Edvinsson - Department of Materials Science and Engineering, The Ångström Laboratory, Uppsala University, SE-75103 Uppsala, Sweden; (1) orcid.org/0000-0003-27597356

Complete contact information is available at:

https://pubs.acs.org/10.1021/acsanm.0c02309

\section{Author Contributions}

G.A.N., A.M.N., and J.W. proposed the idea and the strategy for the experimental design and data analysis. J.W., C.X., Y.H., and H.X. performed the sample preparation, structural characterizations, and optical measurements. T.E. conducted the Raman measurements and data interpretation. J.W. wrote the MATLAB codes. A.M.N. and M.S. contributed to technical discussion and theoretical suggestions on the optical scattering characterization and analysis. J.W. and G.A.N. wrote the paper. G.A.N. supervised the project. All authors discussed the results and commented on the paper.

\section{Notes}

The authors declare no competing financial interest.

\section{ACKNOWLEDGMENTS}

This project was financially supported by the Swedish Research Council grant 2016-03713. M.S. and C.X. acknowledge financial support from the Swedish Research Council Formas grant 221-2012-444. H.X. acknowledges financial support from the China Scholarship Council grant 201708420295 and the Talent Project of Hubei Polytechnic University grant 14xjz03R. The authors are grateful to Daniel Hedlund for the VSM measurements.

\section{REFERENCES}

(1) Mohammed, L.; Gomaa, H. G.; Ragab, D.; Zhu, J. Magnetic Nanoparticles for Environmental and Biomedical Applications: A Review. Particuology 2017, 30, 1-14.

(2) Jeong, U.; Teng, X.; Wang, Y.; Yang, H.; Xia, Y. Superparamagnetic Colloids: Controlled Synthesis and Niche Applications. Adv. Mater. 2007, 19, 33-60.

(3) Zeng, H.; Sun, S. Syntheses, Properties and Potential Applications of Multicomponent Magnetic Nanoparticles. Adv. Funct. Mater. 2008, 18, 391-400.

(4) Hao, R.; Xing, R.; Xu, Z.; Hou, Y.; Gao, S.; Sun, S. Synthesis, Functionalization and Biomedical Applications of Multifunctional Magnetic Nanoparticles. Adv. Mater. 2010, 22, 2729-2742.

(5) Martin, J. E.; Hill, K. M.; Tigges, C. P. Magnetic-Field-Induced Optical Transmittance in Colloidal Suspensions. Phys. Rev. E 1999, 59, 5676-5692.

(6) Patel, R.; Mehta, R. V. Ferrodispersion: A Promising Candidate for An Optical Capacitor. Appl. Opt. 2011, 50, G17-G22.

(7) Mischler, D.; Steinfeld, A. Nonisothermal Nongray AbsorbingEmitting-Scattering Suspension of $\mathrm{Fe}_{3} \mathrm{O}_{4}$ Particles Under Concentrated Solar Irradiation. J. Heat Transfer 1995, 117, 346-354.

(8) Zhao, Y.; Sadat, M. E.; Dunn, A.; Xu, H.; Chen, C.-H.; Nakasuga, W.; Ewing, R.; Shi, D. Photothermal Effect on $\mathrm{Fe}_{3} \mathrm{O}_{4}$ Nanoparticles Irradiated by White-Light for Energy-Efficient Window Applications. Sol. Energy Mater. Sol. Cells 2017, 161, 247-254.

(9) Gorji, T. B.; Ranjbar, A. A. A Review on Optical Properties and Application of Nanofluids in Direct Absorption Solar Collectors (DASCs). Renewable Sustainable Energy Rev. 2017, 72, 10-32.

(10) Lee, I.; Kim, D.; Kal, J.; Baek, H.; Kwak, D.; Go, D.; Kim, E.; Kang, C.; Chung, J.; Jang, Y.; Ji, S.; Joo, J.; Kang, Y. Quasi-Amorphous Colloidal Structures for Electrically Tunable Full-Color Photonic Pixels with Angle-Independency. Adv. Mater. 2010, 22, 4973-4977.

(11) Artoni, M.; La Rocca, G. C. Optically Tunable Photonic Stop Bands in Homogeneous Absorbing Media. Phys. Rev. Lett. 2006, 96, 073905.

(12) Ge, J.; Hu, Y.; Yin, Y. Highly Tunable Superparamagnetic Colloidal Photonic Crystals. Angew. Chem., Int. Ed. 2007, 46, 74287431.

(13) He, L.; Wang, M.; Ge, J.; Yin, Y. Magnetic Assembly Route to Colloidal Responsive Photonic Nanostructures. Acc. Chem. Res. 2012, 45, 1431-1440.

(14) Tang, L.; Casas, J.; Venkataramasubramani, M. Magnetic Nanoparticle Mediated Enhancement of Localized Surface Plasmon Resonance for Ultrasensitive Bioanalytical Assay in Human Blood Plasma. Anal. Chem. 2013, 85, 1431-1439.

(15) Wang, Y.; Dostalek, J.; Knoll, W. Magnetic NanoparticleEnhanced Biosensor Based on Grating-Coupled Surface Plasmon Resonance. Anal. Chem. 2011, 83, 6202-6207.

(16) Huang, S.-H.; Juang, R.-S. Biochemical and Biomedical Applications of Multifunctional magnetic Nanoparticles: a Review. J. Nanopart. Res. 2011, 13, 4411-4430.

(17) Lee, H.; Shin, T.-H.; Cheon, J.; Weissleder, R. Recent Developments in Magnetic Diagnostic Systems. Chem. Rev. 2015, 115, 10690-10724.

(18) Schrittwieser, S.; Pelaz, B.; Parak, W. J.; Lentijo-Mozo, S.; Soulantica, K.; Dieckhoff, J.; Ludwig, F.; Guenther, A.; Tschöpe, A.; Schotter, J. Homogeneous Biosensing Based on Magnetic Particle Labels. Sensors 2016, 16, 828.

(19) Bejhed, R. S.; de la Torre, T. Z. G.; Donolato, M.; Hansen, M. F.; Svedlindh, P.; Strömberg, M. Turn-On Optomagnetic Bacterial DNA Sequence Detection Using Volume-Amplified Magnetic Nanobeads. Biosens. Bioelectron. 2015, 66, 405-411. 
(20) Tian, B.; Ma, J.; Zardán Gómez de la Torre, T.; Bálint, Á.; Donolato, M.; Hansen, M. F.; Svedlindh, P.; Strömberg, M. Rapid Newcastle Disease Virus Detection Based on Loop-Mediated Isothermal Amplification and Optomagnetic Readout. ACS Sens. 2016, 1, 1228-1234.

(21) Howell, J. R.; Menguc, M. P.; Siegel, R. Thermal Radiation Heat Transfer; CRC Press: Boca Raton, FL, 2015.

(22) Prahl, S. A.; van Gemert, J. C.; Welch, A. J. Determining the Optical Properties of Turbid Media by Using the Adding-Doubling Method. Appl. Opt. 1993, 32, 559-568.

(23) Leyre, S.; Leloup, F. B.; Audenaert, J.; Durinck, G.; Hofkens, J.; Deconinck, G.; Hanselaer, P. Determination of the Bulk Scattering Parameters of Diffusing Materials. Appl. Opt. 2013, 52, 4083-4090.

(24) Peters, V. G.; Wyman, D. R.; Patterson, M. S.; Frank, G. L. Optical Properties of Normal and Diseased Human Breast Tissues in the Visible and Near Infrared. Phys. Med. Biol. 1990, 35, 1317-1334.

(25) Che, C.; Lu, J. Q.; Ding, H.; Jacobs, K. M.; Du, Y.; Hu, X.-H. A Primary Method for Determination of Optical Parameters of Turbid Samples and Application to Intralipid Between 550 and $1630 \mathrm{~nm}$. Opt. Express 2006, 14, 7420-7435.

(26) Maheu, B.; Briton, J. P.; Gouesbet, G. Four-Flux Model and A Monte Carlo Code: Comparisons Between Two Simple, Complementary Tools for Multiple Scattering Calculations. Appl. Opt. 1989, $28,22-24$.

(27) Rozé, C.; Girasole, T.; Gréhan, G.; Gouesbet, G.; Maheu, B. Average Crossing Parameter and Forward Scattering Ratio Values in Four-Flux Model for Multiple Scattering Media. Opt. Commun. 2001, 194, 251-263.

(28) Barrios, D.; Alvarez, C.; Miguitama, J.; Gallego, D.; Niklasson, G. A. Inversion of Two-Flux and Four-Flux Radiative Transfer Models for Determining Scattering and Absorption Coefficients for A Suspended Particle Device. Appl. Opt. 2019, 58, 8871-8881.

(29) Kubelka, P.; Munk, F. Ein Beitrag zur Optik der Farbanstriche. Z. Tech. Phys. 1931, 12, 593-601.

(30) Kubelka, P. New Contributions to the Optics of Intensely Light-Scattering Materials. Part I. J. Opt. Soc. Am. 1948, 38, 448-457.

(31) Levinson, R.; Berdahl, P.; Akbari, H. Solar Spectral Optical Properties of Pigments-Part II: Survey of Common Colorants. Sol. Energy Mater. Sol. Cells 2005, 89, 351-389.

(32) Orel, Z. C.; Klanjsek Gunde, M.; Orel, B. Application of the Kubelka-Munk Theory for the Determination of the Optical Properties of Solar Absorbing Paints. Prog. Org. Coat. 1997, 30, 59-66.

(33) Dzimberg-Malcic, V.; Barbaric-Mikosevic, Z.; Itric, K. KubelkaMunk Theory in Describing Optical Properties of Paper (I), (II). Techn. Gaz. 2011, 18, 117-124; 2012, 19, 191-196.

(34) Levinson, R.; Berdahl, P.; Akbari, H. Solar Spectral Optical Properties of Pigments-Part I: Model for Deriving Scattering and Absorption Coefficients from Transmittance and Reflectance Measurements. Sol. Energy Mater. Sol. Cells 2005, 89, 319-349.

(35) Barrios, D.; Vergaz, R.; Sanchez-Pena, J. M.; Granqvist, C. G.; Niklasson, G. A. Toward a Quantitative Model for Suspended Particle Devices: Optical Scattering and Absorption Coefficients. Sol. Energy Mater. Sol. Cells 2013, 111, 115-122.

(36) Wang, J.; Xu, C.; Nilsson, A. M.; Fernandes, D. L. A.; Strömberg, M.; Wang, J.; Niklasson, G. A. General Method for Determining Light Scattering and Absorption of Nanoparticle Composites. Adv. Opt. Mater. 2019, 7, 1801315.

(37) Wang, J.; Xu, C.; Nilsson, A. M.; Fernandes, D. L. A.; Niklasson, G. A. A Novel Phase Function Describing Light Scattering of Layers Containing Colloidal Nanospheres. Nanoscale 2019, 11, $7404-7413$.

(38) Coey, J. M. D. Magnetism and Magnetic Materials; Cambridge University Press: Cambridge, 2010.

(39) Jubb, A. M.; Allen, H. C. Vibrational Spectroscopic Characterization of Hematite, Maghemite, and Magnetite Thin Films Produced by Vapor Deposition. ACS Appl. Mater. Interfaces 2010, 2, 28042812.
(40) Tien, C.-L.; Drolen, B. L. Thermal Radiation in Particulate Media with Dependent and Independent Scattering. Annu. Rev. Heat Transfer 1987, 1, 1-32.

(41) Germer, T. A.; Zwinkels, J. C.; Tsai, B. K. Spectrophotometry: Accurate Measurement of Optical Properties of Materials; Elsevier: Amsterdam, 2014.

(42) Reynolds, L. O.; McCormick, N. J. Approximate TwoParameter Phase Function for Light Scattering. J. Opt. Soc. Am. 1980, 70, 1206-1212.

(43) Haltrin, V. I. One-Parameter Two-Term Henyey-Greenstein Phase Function for Light Scattering in Seawater. Appl. Opt. 2002, 41, $1022-1028$.

(44) Nostell, P.; Roos, A.; Rönnow, D. Single-beam Integrating Sphere Spectrophotometer for Reflectance and Transmittance Measurements versus Angle of Incidence in the Solar Wavelength Range on Diffuse and Specular Samples. Rev. Sci. Instrum. 1999, 70, $2481-2494$.

(45) Rönnelid, M.; Adsten, M.; Lindström, T.; Nostell, P.; Wäckelgård, E. Optical Scattering from Rough-Rolled Aluminum Surfaces. Appl. Opt. 2001, 40, 2148-2158.

(46) Judd, D. B. Fresnel Reflection of Diffusely Incident Light. J. Res. Natl. Bur. Stand. 1942, 29, 329-332.

(47) McPhedran, R. C.; Botten, L. C.; McKenzie, D. R.; Netterfield, R. P. Unambiguous Determination of Optical Constants of Absorbing Films by Reflectance and Transmittance Measurements. Appl. Opt. 1984, 23, 1197-1205.

(48) Bohren, C. F.; Huffman, D. R. Absorption and Scattering of Light by Small Particles; Wiley: New York, 1983.

(49) Schlegel, A.; Alvarado, S. F.; Wachter, P. Optical Properties of Magnetite $\left(\mathrm{Fe}_{3} \mathrm{O}_{4}\right)$. J. Phys. C: Solid State Phys. 1979, 12, 1157-1164.

(50) Querry, M. R. Optical Constants, Report No. AD-A158-623; Missouri Univ.: Kansas City, 1985.

(51) Laven, P. A Computer Program for Scattering of Light from A Sphere Using Mie Theory \& the Debye Series. www.philiplaven.com/ mieplot.htm, 2018 